\title{
Pour une juste appréciation de l'œuvre de Robert Boyle
}

\section{Frédéric Manzini}

\section{(2) OpenEdition \\ 12 Journals}

\section{Édition électronique}

URL : http://journals.openedition.org/etudesirlandaises/2271

DOI : 10.4000/etudesirlandaises. 2271

ISSN : 2259-8863

\section{Éditeur}

Presses universitaires de Rennes

\section{Édition imprimée}

Date de publication : 30 décembre 2011

Pagination : 9-19

ISSN : 0183-973X

\section{Référence électronique}

Frédéric Manzini, « Pour une juste appréciation de l'œuvre de Robert Boyle », Études irlandaises [En ligne], 36-2 | 2011, mis en ligne le 30 septembre 2013, consulté le 20 avril 2019. URL : http:// journals.openedition.org/etudesirlandaises/2271 ; DOI : 10.4000/etudesirlandaises.2271

Ce document a été généré automatiquement le 20 avril 2019.

(c) Presses universitaires de Rennes 


\title{
Pour une juste appréciation de l'œuvre de Robert Boyle
}

\author{
Frédéric Manzini
}

«Mr. Boyle est mort, comme vous saurez déjà sans

doute.

Il paraît assez étrange qu'il n'ait rien bâti sur tant d'expériences dont ses livres sont pleins. »

Christiaan Huygens, Lettre à Leibniz du 4 février $1692^{1}$

1 Si la mondialisation est, avec l'ampleur qu'elle a prise, un processus relativement récent en ce qui concerne l'échange de marchandises, il en va tout autrement pour ce qui touche à la diffusion de la science: depuis longtemps, les idées en ce domaine se propagent aisément et il est rare qu'un penseur connaisse une renommée régionale tout en restant largement méconnu ailleurs. Bien avant d'être des instruments de masse, les moyens de diffusion ont toujours su se mettre à la hauteur des pensées qu'ils avaient pour charge de répandre et, par exemple, la langue n'a jamais constitué une barrière infranchissable : non seulement parce qu'il a toujours existé une langue commune, hier le latin ou le français comme aujourd'hui l'anglais, permettant aux savants de se comprendre quand ils voulaient communiquer entre eux, mais encore parce qu'il n'a jamais été très difficile de trouver des intermédiaires ou des traductions pour prendre connaissance de telle ou telle publication $^{2}$, pour peu que la chose mérite qu'on s'en soit donné la peine. Ainsi, au XVII siècle - puisque c'est ce siècle qui nous retiendra ici - comme aujourd'hui, les idées et les découvertes scientifiques circulent aisément sans connaître le plus souvent de frontières linguistiques ou culturelles ou, plus exactement, en étant ralenties mais non arrêtées par elles : les prétendues «barrières de la langue » n'ont jamais été infranchissables quand l'intérêt éveillé pour un auteur méritait qu'on se donne les moyens de les surmonter.

2 Il existe pourtant quelques exceptions à cette règle, et tel est le cas assez singulier du savant irlandais Robert Boyle (1627-1691). Parce qu'elle a délaissé les anciens principes de l'alchimie au profit de ceux du mécanisme moderne et parce qu'elle a eu recours à une théorie corpusculaire de la matière étayée par de multiples expériences, il est 
incontestable que son œuvre a apporté une contribution majeure à l'histoire de la science en général et à celle de la chimie en particulier. Toutefois, alors que Boyle jouit d'une renommée considérable dans le monde anglo-saxon, où il est tenu pour l'un des hommes de science les plus importants de son époque et pour l'un des penseurs les plus décisifs dans l'infléchissement qu'il sut donner à la pratique scientifique elle-même, sa figure est aujourd'hui largement méconnue et apparaît très marginale au sein de l'Université française. Le fait est d'autant plus étonnant que la France est sans doute le pays continental avec lequel Boyle avait le plus d'attaches : son précepteur lui avait enseigné le français et il séjourna lui-même plusieurs années en Suisse francophone et en France dont il connaît la culture et maîtrise la langue (qu'il utilisait lorsqu'il était à l'étranger). En dépit de ces liens qu'on aurait pu penser privilégiés, aucune traduction française, ou presque, n'a encore été donnée de ses différentes œuvres, pourtant particulièrement nombreuses ${ }^{3}$. À l'exception très notable d'un colloque international d'une certaine ampleur qui s'est tenu en 2005 à l'université de Bordeaux et qui était tout entier dévolu à sa figure ${ }^{4}$, Boyle est absent des recherches et de publications en langue française ${ }^{5}$ : quelques lignes seulement lui sont consacrées dans les histoires de la philosophie visant à l'exhaustivité qui, comme celle d'Émile Bréhier (pour ne prendre que cet exemple) s'étendent sur des milliers de pages.

3 Nous nous proposons ici de tenter d'expliquer la nature et d'interroger la légitimité de ce désintérêt. Pour ce faire, nous nous appuierons sur l'exploration que nous avons menée ${ }^{6}$ d'un certain nombre d'ouvrages, scolaires ou plus érudits, qui sont contemporains ou postérieurs à l'œuvre du savant irlandais, tels qu'ils composent actuellement le fonds de la Bibliothèque Patrimoniale du Collège Irlandais $d u v^{e}$ arrondissement de Paris ${ }^{7}$. Quel meilleur endroit en effet que celui-ci pour évaluer l'écho réel que pouvaient recevoir les thèses de Robert Boyle, grâce à l'examen aussi bien de notes prises à partir des cours professés dans les séminaires britanniques que parmi les publications des savants qui sont proches de lui par la culture, la chronologie et la géographie ? Parce qu'il se situe à l'interface entre la culture britannique et la culture française, l'étude de ce fonds permet en effet de déterminer si l'indifférence manifestée en France à l'égard de Boyle était déjà une réalité à la fin du $\mathrm{XVII}^{\mathrm{e}}$ siècle.

4 À étudier l'ensemble de ces ouvrages et documents de près, on s'aperçoit assez rapidement que, de Boyle, il est dès cette époque très rarement fait mention. Ce constat est facile à établir à partir des cours prononcés dans les séminaires anglais, du moins tels que nous pouvons les connaître d'après les manuscrits qui sont parvenus jusqu'à nous : dans les cours de physique en particulier, nous n'avons guère trouvé de trace qui pourrait indiquer une influence réelle des avancées de Boyle sur le contenu des enseignements qui y sont retranscrits. Même si le nombre de ces manuscrits traitant de physique est restreint dans le fonds de la bibliothèque du Collège irlandais, il est par exemple significatif que le manuscrit $n^{\circ} 64$ ne cite pas Boyle parmi les physiciens récents mais seulement Galilée, Gassendi et Descartes ${ }^{8}$. Il en va de même pour la Physica retranscrite dans le manuscrit $n^{\circ} 149$ qui se réfère à Descartes ${ }^{9}$, Newton ${ }^{10}$, Berkeley ${ }^{11}$, Robert Hooke ${ }^{12}$ et même Zénon ${ }^{13}$, mais oublie de mentionner le savant irlandais. C'est également le cas de la plupart des œuvres imprimées: ainsi, par exemple, la partie "physique » de la Philosophia Maignani scholastica du minime Jean Saguens, publiée en 1703, n'étudie successivement que quatre écoles au moment d'envisager les principes de la physique: " ad mentem Aristotelis » puis « ad mentem cartesii » puis « ad placitum gassendi » et enfin " ad sententiam Patris Maignani ${ }^{14}{ }^{4}$. Plus surprenant sans doute, Boyle n'est, sauf omission, pas 
davantage mentionné dans un ouvrage comme le Scepsis Scientifica, or the vanity of dogmatizing de Joseph Glanvill, publié en 1665. On aurait pourtant pu s'attendre à ce qu'il le soit: non seulement parce que Joseph Glanvill est personnellement très proche de Robert Boyle, mais encore parce qu'il adresse son ouvrage à la Royal Society dont Boyle est le membre le plus éminent et enfin parce que le contenu même du volume - dont le titre rappelle suffisamment celui du décisif Sceptical Chemist de Boyle paru à peine quelques années plus tôt - est très semblable aux thèses développées par celui-ci, notamment en ce qui concerne le rôle du savant et la défiance dont il doit faire preuve vis-à-vis des systèmes prétentieux et des spéculations trop théoriques. En dépit de ces affinités évidentes, Joseph Glanvill ne se réfère qu'à Kenelm Digby, Descartes, Henry More et aux Platoniciens ${ }^{15}$ (c'est-à-dire aux Platoniciens de Cambridge) quand il considère les doctrines modernes qui cherchent à expliquer la nature du lien entre l'âme et le corps ; quand il examine les théories concernant la mémoire, il envisage de la même manière «the Aristotelian, Cartesian, Digbæn and Hobbian Hypothesis »; et c'est toujours ainsi qu'il procède. Alors que Descartes est l'auteur de référence de Joseph Glanvill ainsi que son principal interlocuteur, et tandis qu'Aristote apparait comme son plus grand adversaire, Boyle, en revanche, reste absent.

Le désintérêt dont la figure de Robert Boyle souffre actuellement en France (et, d'une manière plus générale, sur l'ensemble du continent européen non-anglophone) n'est donc peut-être pas si nouveau qu'il le semble et pourrait même remonter au XVII siècle. Comment l'expliquer ? Cinq facteurs méritent, à notre sens, d'être mis en avant.

6 1) Le premier d'entre eux vient du fait que Boyle soit essentiellement un physicien et un chimiste. En tant que tel et à une époque où le progrès scientifique était déjà rapide, un savant était exposé plus qu'un autre à être dépassé par des collègues plus innovants. Or ceci est d'autant plus vrai dans le cas de Boyle qu'il n'a pas apporté de découverte originale majeure à laquelle son nom pourrait être associé ${ }^{16}$. De fait, sa renommée eut très vite à souffrir outre-Manche de la concurrence du grand Isaac Newton (1643-1727), qui ne lui est postérieur que d'une quinzaine d'années mais dont l'importance de l'œuvre est évidemment si considérable qu'elle n'a pas tardé à intégrer, à dépasser et finalement à éclipser celle de Boyle. En France, le désintérêt des auteurs pour l'œuvre de Boyle peut en outre s'expliquer par le fait que le pays disposait déjà avec Descartes et Gassendi de ses propres champions de la philosophie mécaniste ${ }^{17}$.

7 2) Les circonstances historiques dans lesquelles les travaux de Boyle ont été reçus en France sont un deuxième facteur pour expliquer le manque d'intérêt suscité par sa pensée. On sait qu'à la fin des années 1660 l'Académie des Sciences de Paris (dont on peut considérer qu'elle représente en France le pendant de la Royal Society anglaise) s'est montrée suffisamment intéressée par les travaux du savant irlandais pour commander au chimiste français Samuel Duclos ${ }^{18}$ un compte rendu détaillé : aussi Duclos fit-il, lors de treize sessions de l'Académie étalées entre la fin de l'année 1668 et le début de 1669 une analyse critique des thèses de Boyle. Or les conclusions de ces sessions se sont avérées suffisamment défavorables, non seulement sur les capacités techniques de Boyle mais également sur sa doctrine proprement dite et notamment sur le principe même d'une chimie corpusculaire - jugement que, rétrospectivement, on s'autorisera à trouver injuste ! - pour décourager un certain nombre de savants français d'aller approfondir leur connaissance de ses travaux afin de se forger leur propre opinion. Dès lors, on comprend mieux le dépit avec lequel Lawrence Principe constate qu'« en dehors de Duclos, peu de chimistes français réagirent explicitement aux théorisations de Boyle ${ }^{19} »:$ seuls quelques 
rares savants de second ordre lui ont montré un certain intérêt mais, de son vivant déjà, il n'était pas en France un savant très réputé.

8 3) Une troisième raison repose sur la manière dont Boyle a été perçu chez ceux qui néanmoins le mentionnent. Le meilleur relais du physicien philosophe en France est probablement Jean-Baptiste Du Hamel, secrétaire de l'Académie Royale des Sciences. De fait, dans la partie Physica de l'édition de 1682 de sa remarquable Philosophia vetus et nova, Du Hamel fait plusieurs fois référence au traité de Boyle intitulé Le Chimiste sceptique à l'appendice duquel il renvoie à propos des différentes sortes de nitre ${ }^{20}$. Suivent plusieurs autres références aux expérimentations de Boyle dans le chapitre "De mixtis perfectis, seu de fossilibus » de cette Physique, qui se révèlent le plus souvent être de sincères éloges adressés au chimiste irlandais ${ }^{21}$ : ce dernier n'est certes pas le seul physicien cité puisque son nom côtoie ceux d'un grand nombre d'autres savants du XVII e siècle aussi divers que Pierre Borel, Samuel Duclos, Nehemiah Grew, Pierre de Carcavi, Philippe de La Hire, Jean Gallois et José de Acosta par exemple. Mais aux yeux de Du Hamel, Boyle se distingue nettement des autres savants : «sed praeter caeteros D. Boyle hoc argumentum diligenter, \& docte tractasse mihi videtur ${ }^{22}$. » Quelle que soit la reconnaissance que manifeste Du Hamel à l'endroit de Boyle, il n'en demeure pas moins que celui-ci ne semble donc intéresser celuilà que pour les expérimentations chimiques qu'il a menées. Même un savant particulièrement favorable à Boyle comme l'est manifestement Du Hamel ne mentionne ainsi l'auteur irlandais que dans un domaine assez limité où il reste cantonné.

Le cas est en réalité révélateur d'une attitude plus générale : au mieux, Boyle est toujours apparu comme un faiseur d'expériences ${ }^{23}$ plus que comme un savant, comme un technicien capable d'enregistrer des éléments factuels mais non comme un philosophe véritable qui aurait une doctrine et une pensée théorique personnelle forte. Lui-même aurait sans doute volontiers souscrit à ce constat par l'importance qu'il accorde aux expériences dans la démarche scientifique, au point d'en faire le cœur même de sa méthode. Plus que les résultats obtenus proprement dits, c'est d'ailleurs ce caractère radicalement expérimental de sa pensée qui doit assurément être considéré comme la marque la plus forte de l'originalité de Boyle, par laquelle il se démarque nettement des autres savants. Mais cette singularité n'a pas toujours été portée à son crédit, notamment de la part des penseurs rationalistes qui, surtout pendant le "grand siècle", font davantage confiance à leur esprit qu'à leurs sens, et à la théorie qu'à l'expérience, pour faire progresser le savoir.

10 Le fait que Boyle se soit toujours refusé à donner à sa philosophie l'unité d'un tout, autrement dit son refus viscéral de l'esprit de système ${ }^{24}$, l'a conduit à présenter ses idées de manière très éclatée dans des Traités multiples et divers ; or ce choix revendiqué rend, aujourd'hui encore, la tâche bien difficile à celui qui voudrait appréhender sa pensée dans sa cohérence générale. Ses contemporains déjà, tout en reconnaissant la valeur de ses découvertes et son habileté dans les expérimentations, s'étonnaient et s'agaçaient de cet éparpillement et du zèle déployé par Boyle pour ne pas faire la synthèse de sa science dans ce qui aurait pu constituer un remarquable système de chimie : c'est notamment le cas de Leibniz, qui avait plusieurs fois insisté vainement auprès d'Oldenburg pour qu'il incite son ami à remédier à cette lacune l'empêchant de porter un jugement global sur ses travaux. Chacun de leur côté, Spinoza, Günther Christoph Schelhammer et Hobbes notamment déploraient également l'incapacité de Boyle à raisonner au-delà de l'expérience ${ }^{25}$. Il faut avouer que le savant irlandais n'a jamais rien fait pour démentir cette réputation, au contraire. Refusant obstinément de théoriser au-delà du strict 
nécessaire, il semble inévitable que Boyle soit resté aux yeux de nombreux philosophes comme un simple expérimentateur sans envergure véritable: loin de se prétendre le savant universel, n'accepte-t-il pas volontiers lui-même d'endosser le rôle d'un drudge, autrement dit d'un simple et laborieux tâcheron qui se place humblement au service du progrès du savoir ${ }^{26}$ ? Toujours est-il qu'il a dû moins apparaître aux yeux d'un certain nombre de ses contemporains comme un auteur de référence aux positions clairement identifiables que comme un habile fournisseur de matériaux scientifiques dans lesquels on peut puiser et qu'on peut utiliser pour leur donner un sens original (sans qu'il soit pour autant nécessaire de saluer ni même, éventuellement, de mentionner celui qui les a mis au jour). Faut-il regretter une prudence excessive ou faut-il au contraire féliciter Boyle d'avoir su résister à la pression de ses pairs et d'être resté toujours modeste et conséquent avec ses propres principes?

11 L'appréciation qui sera portée sur son attitude dépend sans doute de celui qui énonce le jugement. D'une manière générale en effet, le chimiste irlandais apparaît comme un cas très représentatif de l'écart qui sépare l'esprit de la philosophie française (mais aussi allemande), profondément marquée par le rationalisme spéculatif de type cartésien, et la tradition britannique, qui hérite au contraire de l'empirisme de type baconien. Or Boyle se voit très volontiers comme le sectateur de Francis Bacon de ce point de vue, même s'il a été attiré par le cartésianisme d'un autre point de vue. On comprend donc très bien qu'à cet égard ce terreau de la philosophie fondée sur l'expérience qu'est l'Angleterre fut, et continue à être, autrement plus réceptif à la science de Boyle que ne le furent l'Europe continentale en général et la France en particulier, où tout ce qui apparaît comme un tant soit peu empiriste a tôt fait de se trouver méprisé comme quelque chose de vulgaire.

12 4) Une quatrième raison permet d'expliquer la méfiance dont, particulièrement en France, la pensée de Boyle peut être l'objet dès son époque et davantage encore aujourd'hui : il s'agit du souci presque constant que le savant affiche, y compris et même surtout dans ses écrits proprement scientifiques, pour des questions d'ordre proprement théologique ou religieux. En plus d'être un érudit, Boyle est en effet un apologiste convaincu et vigoureux, qui fait volontiers le lien entre la "vérité » scientifique et la "vérité " chrétienne. Or là encore, la manière de penser française répugne particulièrement à cette volonté de mêler intimement ces deux registres de l'exactitude scientifique et du prosélytisme religieux. Il est aisé de comprendre que la façon qu'a Boyle de mettre la science et les savants au service de la défense de la religion chrétienne et d'un combat partisan contre l'athéisme - qui se retrouve évidemment dans le principe même des Boyle lectures - ait de quoi éveiller toutes les suspicions dans un pays dont on sait à quel point il s'identifie parfois au principe d'une laïcité dont il a fait depuis le début $\mathrm{du} \mathrm{xx}^{\mathrm{e}}$ siècle l'une des valeurs absolument fondamentales de sa république ${ }^{27}$. Or rien n'est plus étranger à Boyle que cette volonté de séculariser la science: ce "physicien théologien » ou ce "théologien physicien " qu'est Boyle tient constamment à lier son naturalisme à ses convictions religieuses, comme en témoigne aussi ce passage de l'adresse « au lecteur » des Quelques considérations concernant le style des Écritures saintes :

Je t'avoue également qu'à cause des écrits physiologiques que j'ai été amené à publier dernièrement et du genre d'études à laquelle je semble me consacrer exclusivement à présent (pour des raisons qui devront être précisées en temps voulu), de nombreuses personnes me considèrent comme un naturaliste. Et certains, aussi bien des philosophes que des physiciens ont, soit par faute soit par négligence, fourni l'occasion à de nombreux hommes de penser que ceux qui étudient de manière spéculative les mystères de la nature s'appuient, comme je le 
fais souvent, sur la philosophie péripatéticienne commune, et en particulier ceux qui sont favorables à l'explication des phénomènes de la nature par les atomes, tendent vers l'athéisme, ou au moins tendent à n'être concernés par aucune religion que ce soit. Puisqu'il en va comme je l'ai dit, je ne suis pas mécontent de saisir cette occasion de témoigner publiquement d'une façon qui pourra rassurer ceux qui ne me connaissent pas (car je suppose que ceux qui me connaissent bien sont déjà rassurés) : même si je suis un naturaliste, il est possible de l'être sans être ni un athée ni quoi que ce soit de ce genre. Et l'étude des œuvres de la nature ne m'a pas fait cesser de croire en leur Auteur, ni nier sa Providence, ni même mépriser son monde, qui mérite notre respect à plusieurs titres, et en particulier au titre qu'il est le grand instrument qui nous transmet les vérités et les mystères de la religion chrétienne ${ }^{28}$.

13 Boyle veut non seulement montrer qu'il est possible d'être naturaliste sans être athée, mais il estime même qu'il est nécessaire de postuler l'existence d'une cause première divine qui seule est susceptible de rendre raison de tous les phénomènes de la nature ${ }^{29}$. Aussi le savant déclare-t-il dans les premières lignes de son ouvrage Christian Virtuoso publié en 1690 que «la philosophie expérimentale mène à la religion entendue d'une manière générale »: le spectacle de l'univers dans son admirable complexité, affirme-t-il, ainsi que la constance des lois qui le régissent ne sauraient s'expliquer par la doctrine matérialiste et doivent faire conclure à la nécessité d'un auteur intelligent. Robert Boyle a ainsi cherché à montrer la compatibilité et même la continuité supposées exister à ses yeux entre la religion (chrétienne) d'une part et la science d'autre part. Là encore cependant, compte tenu du fossé fondamental qui sépare sur ce point la culture britannique (à plus fort titre la culture américaine) et la culture française, on comprend aisément qu'un tel projet ait reçu deux échos bien différents de part et d'autre de la Manche. Par comparaison, un philosophe comme Locke qui s'est fait au contraire le défenseur de la liberté de conscience et d'une tolérance entendue comme indifférence vis-à-vis de la question religieuse aura reçu en France un accueil des plus bienveillants dès le XVIII ${ }^{\mathrm{e}}$ siècle.

14 5) Enfin, on peut ajouter aux raisons précédemment avancées le fait que l'influence réelle de la pensée de Boyle soit peut-être artificiellement surévaluée outre-Manche à cause de - ou plutôt grâce à - l'empreinte que l'homme et le citoyen, plus que le savant, ont pu laisser au sein de la communauté scientifique et, au-delà, de la société civile anglaise ellemême. Outre son intense activité pendant les années qu'il a passées à la Royal Society de Londres dont il fut l'un des fondateurs et sans doute la personnalité la plus influente, il faut en effet évoquer le rôle exercé par les Boyle Lectures qu'il a initiées par disposition testamentaire : ces conférences, qui continuent aujourd'hui encore de se tenir de manière annuelle, sont vite devenues une véritable institution parmi les personnalités scientifiques et se sont avérées dès 1691 si importantes pour la communauté des savants dans le monde anglo-saxon, qu'on peut raisonnablement penser qu'elles ont également fortement contribué à gonfler une renommée finalement plus civile que scientifique à proprement parler. Même si Boyle n'est pas lui-même responsable du contenu de ces interventions dont il n'a fait que fixer l'orientation en réclamant qu'elles servent à promouvoir la religion chrétienne, le prestige des différents intervenants n'a pu indirectement qu'ajouter au sien, tout simple mécène qu'il soit en la circonstance.

Il y a donc un certain nombre de raisons, plus ou moins rédhibitoires mais qui semblent toutes assez tenaces, susceptibles d'expliquer que Robert Boyle soit resté dans l'ombre de l'histoire de la philosophie et que rares furent les érudits qui se soient intéressés à ses travaux. La situation peut-elle évoluer à plus ou moins longue échéance? Si les cinq 
raisons que nous avons mises en avant sont toujours d'actualité, elles pourraient inciter à croire que la pensée de Boyle va continuer à rester minorée dans les études universitaires françaises et, plus généralement, continentales. Mais les choses peuvent également changer, notamment en vertu du retournement de la troisième des raisons que nous avons mentionnées. Ce qui en effet lui était implicitement ou explicitement reproché à son époque pourrait bien mériter aujourd'hui un intérêt particulier au point de devenir au contraire tout ce qui en fait la force: la défiance généralisée à l'égard des systèmes prétentieux trop théoriques qu'affiche Boyle, ainsi que son souci obsessionnel de la pratique scientifique et de la vérification expérimentale, n'étaient-ils pas précurseurs de "l'ère du soupçon" dans laquelle nous sommes à ce jour? Ce qui passait pour de la pusillanimité et de l'étroitesse de vues à son époque ne se révèle-t-il pas, à l'usage, être la marque d'une sage prudence qui a beaucoup à nous apprendre sur la nécessaire modestie des scientifiques? Avec le recul dont nous disposons et un regard rétrospectif, il semble bien que nous soyons davantage capables aujourd'hui de mieux apprécier la véritable portée de la pensée de Boyle, ce dont la réédition récente de ses œuvres complètes par Michael Hunter et Edward B. Davis ${ }^{30}$ ainsi que les publications collatérales qui l'accompagnèrent ${ }^{31}$ sont à la fois un effet et une cause. Il apparaît important désormais, y compris et surtout en France, de réévaluer et de redécouvrir son œuvre, pour lui rendre enfin justice.

\section{NOTES}

1. CEuvres complètes de Christiaan Huygens, Société Hollandaise des sciences (éd.), La Haye, 1888-1950, t. X, p. 239.

2. Il suffit de penser précisément à la façon dont Boyle et Spinoza ont pu entretenir un échange suivi et précis grâce au truchement de Henry Oldenburg de la Royal Society.

3. Seuls deux essais, qui ne sont malheureusement pas les plus intéressants et s'avèrent assez techniques, ont été traduits. Le premier, "Sur les saveurs et les odeurs", a été inséré dans un collectif intitulé Recueil d'expériences et observations sur le combat qui procède du mélange des corps par N. Grew, sur les saveurs, sur les odeurs par R. Boyle, sur le sang, sur le lait, etc. par A. Van Leeuwenhoek (Paris, E. Michallet, 1679) et le second est le Nouveau traité de Monsieur Boyle sur la convenance des remèdes spécifiques de la philosophie des corpuscules, \& sur l'usage \& les propriétés des médicaments simples (trad. fr. J. de Rostagny, Lyon, Jean Certes, 1689). Depuis plus de trente ans a paru pourtant une traduction italienne de quelques-unes de ses œuvres majeures: Robert Boyle, Opere, a cura di Clelia Pighetti, Torino, 1977.

4. Les actes de ces journées ont été publiés par Myriam Dennehy et Charles Ramond (dir.), La philosophie naturelle de Robert Boyle, Paris, Vrin, 2009.

5. Il serait évidemment exagéré de considérer que Boyle est toujours resté totalement marginalisé au sein des études universitaires. Il n'en demeure pas moins qu'il n'a jamais été au centre des débats scientifiques comme philosophiques et cette situation dure jusqu'à aujourd'hui dans les travaux d'histoire des sciences et de philosophie. En France, 
on ne s'y intéresse souvent qu'à cause de Spinoza et à travers lui, parce qu'il existe toute une correspondance, suivie et précise, qui indique que Spinoza a lu de près les Certain physiological essays de 1661 par l'intermédiaire du secrétaire de la Royal Society Henry Oldenburg : c'est le cas de Pierre Macherey dans son article «Spinoza lecteur et critique de Boyle » (dans « La communication entre savants dans l'Europe du Nord-Ouest de 1660 à 1740 ", Revue du Nord, t. LXXVII, octobre-décembre 1995, p. 733-774) et de Charles Ramond (voir la note précédente et l'article qu'il cosigne avec Evelyne Guillemeau dans ce volume). C'est également notre cas : Frédéric Manzini, «Verum index sui : de Spinoza à Boyle (à Aristote)», Archives de philosophie, 73/4, oct.-déc. 2010, Bulletin de bibliographie spinoziste, XXXII, p. 741-744.

6. Nous avons pu mener à bien cette recherche dans des conditions confortables permises par l'une des bourses que, depuis quelques années, le Centre Culturel Irlandais offre à des chercheurs qui se proposent de travailler sur leur fonds ancien. Nous profitons de l'occasion qui nous est ici offerte pour exprimer notre gratitude pour la confiance qui nous a été accordée pendant notre recherche.

7. Le fonds qui est aujourd'hui celui de la bibliothèque n'est malheureusement pas la collection historique d'origine de l'Irish College puisque celle-ci fut perdue au cours de la Révolution française. Mais la collection actuelle - qui compte environ huit mille ouvrages, dont près de la moitié a été publiée entre le $\mathrm{xv}^{\mathrm{e}}$ et le $\mathrm{xVIII}{ }^{\mathrm{e}}$ siècle - regroupe des livres et des manuscrits provenant d'établissements religieux anglophones dissous, tels que le Collège des Écossais et le Séminaire anglais à Paris. Nous précisons entre chevrons les cotes des ouvrages de ce fonds quand nous les citons dans le présent article.
8. $<\mathrm{MS} 64>$, p. 2.
9. $<$ MS 149>, passim.
10. Idem, p. 16.
11. Idem, p. 24.
12. Idem, p. 43-44.
13. Idem, p. 34.

14. $<$ C 298 (1) > = Philosophia Maignani scholastica, Tolosae, A. Pech, 1703, vol. I, t. II [Physica], disp. I, art. I-IV, p. 3-47.

15. <B 438> Scepsis Scientifica, London, E. Cotes for H. Eversden, 1665, p. 12 sq.

16. On retient souvent de Boyle la loi de thermodynamique appelée «loi de Boyle » ou " loi de Boyle-Mariotte », et accessoirement la mise au point de la pompe à air.

17. Encore faudrait-il définir précisément ce qu'on entend par " mécanisme » car il n'est pas certain que Descartes et Gassendi soient aussi mécanistes que Boyle ou le soient comme lui, qui est peut-être le premier véritable philosophe mécaniste - mais cette question devrait faire l'objet d'une étude spécifique.

18. Samuel Cotterau Du Clos ou Cotreau-Duclos (1598-1685), qui fut aussi médecin ordinaire du roi. Voir aussi les écrits de Fontenelle (selon lequel c'est Nicolas Lémery qui représenterait le premier chimiste rationnel) relatant les travaux de l'Académie des Sciences pour l'année 1669.

19. Lawrence M. Principe, «Liens et influences chimiques entre Boyle et la France », dans C. Ramond et M. Dennehy (dir.), La philosophie naturelle de Robert Boyle, op. cit., p. 83.

20. "Hinc optimè animadverterit D. Boyle in appendice ad Chimistam scepticum nuper edita locum illum proverb. 25. Acetum in nitro, qui cantat Carmina cordi pessimo, hoc est afflicto, ut est 
in Hebraeo ; intelligi non posse de vulgari nitro, seu sale petrae, qui, cum aceto non tumultuatur; sed optimè explicari de nitro Ægyptiaco, quod nunc natrum vocant. Nam sal ille lixivialis cum aceto permistus effervescit 》 (<B 1260b $>$ = Philosophia vetus et nova, Physica, Noribergae, J. Ziegeri, 1682, pars II, tract. 3, cap. 2, p. 476) puis à la page suivante : «Cum enim ut fusè explicat D. Boyle in appendice nuper laudata, salpetrae in acervum pyramidi aut cono similem congestus \& aeri expositus accensi carbonis ope paulatim incenditur, \& ex 16, uncis decem salis fixi \& lixivialis hac arte eliciuntur, textura tantummodo partium immutatur, tum ex salpetrae fit nitrum fixum sali tartari consimile» (idem, p. 477). Est-ce une coïncidence ? Cette question du nitre et du salpêtre est précisément celle à propos de laquelle Spinoza a entretenu une correspondance savante avec Boyle.

21. "Refert D. Boyle loco citato fel vitri, seu salem, qui ex materia vitri fusa cochleari ferreo extrahitur, postquam in aqua pura exsolutus, \& filtratus, tum humore superfluo identitem exhalato, in frigidiore loco repositus primum christallos puras \& pellucidas, tum grana perpolita salis communis praebuit » (idem, p. 478); «Itaque volatilitas, ut fusè \& ingeniosè explicat D. Boyle in peculiari tractatu, ex affectionibus mechanicis oritur» (idem, p. 480); "Multa in hanc rem exempla affert D. Boyle fuit illi adamas rudis, qui vel digito pressus, \& levi motus affrictus, saepe etiam cum è pera subduceretur, citra ullam affrictionem vim suam electricam expromebat » (idem, p. 486); "Deinde in machina pneumatica exhausto aere succinum paleas ad se rapit, ut expertus est D. Boyle, \& tamen nullus est hic locus atmosphaera aeris" (idem, p. 488); "sed praeter caeteros D. Boyle hoc argumentum diligenter, \& docte tractasse mihi videtur » (idem, p. 491) ; «Sic D. Boyle se vidisse testatur lapidem chrystallo similem \& exquisitè figuratum, qui circa filum metallicum \& ramosum, quod purum fuisse argentum existimabat, concreverat " (idem, p. 493) ; "Inter alia quae in hanc rem affert D. Boyle, ut probet gemmas si minus alterari in ventriculo, certe vires suas exerere, illud refert memorandum, penes se fuisse adamantem, qui in aqua calida positus in tenebris luceret» (idem, p. 494); "Cum autem teste Boylio marmoris albi gravitas adaquae gravitatem sit ferè [...]" (idem, p. 495-496); "Hujus occasione elegantem tractatum conscripsit de solido in solido, ubi iisdem ferè principiis utitur, quibus D. Boyle, licet eodem ferè tempore scripserint. [...] Interalia plurima D. Boyle silicem asservat, qui serpentem exquisitè formatum \& convolutum, sed sine sapite, continet, atque hic serpens ex alio genere lapidis \& limpidiore constat » (idem, p. 496). Dans les pages qui suivent, les références à Boyle se font plus rares: "Aut quod idem testatur Boylius, cum magnes ignitus vim suam magna ex parte exuit, \& polos commutat [...]» (idem, p. 502) ; «in ampla retorta exstillat D. Boyle» (idem, p. 512) ; "Haec confirmari possent ex iis, quae D. Boyle tractatu de lantentibus quibusdam aeris qualitatibus feusè expicat de lapide duro \& subnigro » (idem, p. 516). On trouve aussi quelques allusions à Boyle dans d'autres chapitres, comme par exemple celle-ci : "Unum cupri granum, ut Clariss. Boylius peculiari tractatu de Effluviis docet, spiritu salis armoniaci dissolve, fiet liquor coeruleus » (idem, p. 133) mais elles semblent isolées et très ponctuelles.

22. « Mais par rapport aux autres, Boyle me semble avoir traité cet argument de manière consciencieuse et savante " (Philosophia vetus et nova, Physica, op. cit., p. 491, déjà citée dans la note précédente). Du reste, l'estime cordiale que Du Hamel porte à Boyle semble avoir été payée en retour par celui-ci comme en atteste un paragraphe ajouté à titre d'appendice à cette édition de la Philosophia vetus et nova affirmant : "Cum haec typis jam mandata essent, literas à nobilissimo Viro D. Boyle accepimus plenas humanitatis, quibus nos certiores facit, se tractatum de phosphoris edidisse, quem ad nos mittere cum nondum posset, interim viam \& rationem qua utrumque phosphorum tum siccum, tum acetum in suo laboratorio parasset, benigne nobiscum communicat » (Philosophia vetus et nova, op. cit., " appendix ad pag. 705 De Phosphoris », p. 843). 
23. C'est également ainsi que le considère Jean Saguens dans l'une des rares mentions qu'il fait du nom de Boyle : "Ad id vero quod additur, habeatne ignis suum pondus in mixtis, in quibus substantaliter, continetur, affirmative respondeo \& moveor, praeter rationem, quae suadet pondus totale mixti coalescere ex ponderibus partialibus singulorum miscibilium, quamplurimis experimentis quae fecit Boylius circa ponderabilitatem flammae \& ignis, \& apud ipsum legi poterunt. Unde in hoc sensu ignis est gravis» (<C 298 (2)> = Philosophia Maignani scholastica, vol. II, t. III, disp. XIII, art. II [De mobilitate ignis], § XII, p. 320).

24. Voir l'Essai préliminaire qui est le premier des Certain Physiological Essays, London, H. Herringman, $1661(=<$ B795 $>)$ et dont nous publierons bientôt une traduction originale en français.

25. Voir Myriam Dennehy, «Leibniz et Sturm, lecteurs de Boyle» dans C. Ramond et M. Dennehy (dir.), La philosophie naturelle de Robert Boyle, op. cit., notamment p. 334-335, qui détaille plus qu'il ne nous est utile ici le rapport de ces différents auteurs à Boyle. Contentons-nous de citer Leibniz qui, après avoir souligné le "grand talent» du " chevalier Boyle » pour mener les expérimentations, ajoute en citant et en approuvant Spinoza que Boyle « s'arrête un peu trop, pour dire la vérité, à ne tirer qu'une infinité de belles expériences d'autre conclusion que celle qu'il pouvait prendre comme principe, savoir que tout se fait mécaniquement dans la nature, principe qu'on peut rendre certain par la seule raison, et jamais par les expériences, quelque nombre qu'on en fasse.» ( Nouveaux Essais sur l'entendement humain, livre IV, chap. 12, § 13).

26. Voir à nouveau l'Essai préliminaire, op. cit., p. 17 de l'édition originale de 1661.

27. Il existe certes en France des philosophes ouvertement apologistes comme Pascal par exemple. Mais ce qu'on pardonne assez volontiers à un « littéraire » est sans doute moins facilement acceptable de la part d'un "scientifique» dont on attend objectivité et impartialité. Isaac Newton (qui sur ce point prolonge tout à fait la perspective mécanicofinaliste de Boyle) constitue cependant une exception remarquable, qui s'explique sans doute par l'importance de ses découvertes.

28. $\angle \mathrm{B}$ 805> = Robert Boyle, Some considerations concerning the style of the Holy Scriptures, London, H. Herringman, 1663, « To the reader », p. C2v.

29. Voir par exemple Robert Boyle, An Essay containing a requisite digression concerning those that would exclude the Deity from intermeddling with matter, dans Some Considerations touching the usefulness of experimental natural philosophy, vol. IV, Oxford, 1663.

30. The Works of Robert Boyle, M. Hunter et E. Davis (éd.), London, Pickering \& Chatto, 2000-2001.

31. Voir The Correspondence of Robert Boyle, M. Hunter, A. Clericuzio et L. Principe (éd.), London, Pickering \& Chatto, 2001, 6 vol., ainsi que, parmi les publications récentes, Michael Hunter, Boyle: Between God and Science, New Haven, Yale University Press, 2009. 


\section{RÉSUMÉS}

Pour quelles raisons l'importance de l'œuvre du chimiste et philosophe irlandais Robert Boyle (1627-1691) a-t-elle toujours été sous-estimée dans un pays comme la France? Le savant est pourtant tenu dans le monde anglo-saxon pour l'un des penseurs les plus décisifs de la science moderne, notamment par la nouvelle méthode expérimentale dont il s'est servi pour assurer définitivement le triomphe du mécanisme. Cet article examine cinq facteurs permettant d'expliquer cette marginalisation continentale, depuis les conditions dans lesquelles les découvertes de Boyle ont été dès son époque reçues par ses pairs, jusqu'à la différence d'approche, en France et dans les pays anglophones, du rapport entre science et religion.

Robert Boyle's scientific and philosophical writings have always been neglected in France in particular. Whereas the latter is often held in Ireland and in England to be one of the major scientists and scholars of modern ages - especially because of the new experimental method he used in order to promote mechanism - he is usually relegated to a position of secondary importance in the country of Descartes. The article puts forward five explanations for the double standard, from historical perspectives concerning the context of the reception of his works in the 17th century to the difference of points of view concerning the relationships between science and religion.

\section{INDEX}

Mots-clés : science, philosophie, relations franco-irlandaises, religion, Boyle Robert

Keywords : philosophy, Franco-Irish relations

\section{AUTEUR}

\section{FRÉDÉRIC MANZINI}

Université Paris IV 